論文

스트랩다운 탐색기를 장착한 전술유도탄의 UKF 기반 종말호밍 유도 오승민*

\title{
Terminal Homing Guidance of Tactical Missiles with Strapdown Seekers Based on an Unscented Kalman Filter
}

Seung-Min Oh*

\begin{abstract}
Recent development in seeker technology explores a new seeker design in which, with larger field-of-view (FOV), optical parts are strapped down to a body (hence, called as a body-fixed seeker or a strapdown seeker). This design has several advantages such as comparatively easier maintenance and calibration by removing complex mechanical moving parts, increasing reliability, and cost savings. On the other hand, the strapdown seeker involves difficulties in implementing guidance laws since it does not directly provide inertial LOS rates. Instead, information for generating guidance commands should be extracted by estimating missile/target relative motion utilizing target images on the image plane of a strapdown seeker. In this research, a new framework based on an unscented Kalman filter is developed for estimating missile/target relative motion on the simplified assumption of a point source target. Performance of a terminal guidance algorithm, in which guidance command is generated based on the estimated relative motion, is demonstrated by a missile/target engagement simulation.
\end{abstract}

\section{초 록}

일반적인 전술유도탄에서 표적획득 센서로 사용되는 김발형 탐색기(Gimballed Seeker) 는 탐색기의 표적획득부가 유도탄의 동체운동과는 독립적으로 관성좌표계 상에서 표적을 지향하게 하여 비례항법 유도명령 생성에 필요한 유도탄/표적간의 시선 각속도 정보를 직접 제공한다. 반면 최근에는 탐색기 전체가 유도탄 동체에 고정됨으로써 정비보수 용 이, 고장수리 감소, 단가감소 측면 등에서 유리한 동체고정 탐색기(Strapdown Seeker)에 대한 연구가 부분적으로 수행되고 있다. 그런데 동체고정 탐색기에서는 비례항법에 필요 한 시선각속도가 직접주어지지 않고 탐색기 영상 평면에 맺힌 표적영상으로부터 유도명 령생성에 필요한 정보를 추출하여야 한다. 본 연구에서는 먼저 스트랩다운 탐색기로부터 주어지는 표적정보를 바탕으로 언센티드 칼만필터(Unscented Kalman Filter, UKF)를 적 용하여 유도탄과 표적간의 상대운동을 추정한다. 추정된 상대운동 정보를 이용하여 유도 명령을 생성하고 유도탄과 표적간의 조우운동을 시뮬레이션을 통하여 검증하였다.

Key Words : Strapdown Seeker(스트랩다운 탐색기), Terminal Guidance(종말유도), Unscented Kalman Filter(언센티드 칼만필터)

†2009년 12월1일 접수 2010년 1월17일 심사완료

* 정회원, 국방과학연구소 제 1 기술연구본부 2 부

교신저자, E-mail : seungt@gmail.com

대전광역시 유성우체국 사서함 35-12호 


\section{I. 서 론}

발사후 망각(Fire-and-forget) 방식의 대부분의 단거리 전술유도탄들은 주야간 교전능력을 확보 할 수 있도록 적외선 탐색기를 장착하고 있다. 적외선 탐색기로부터 획득된 표적정보를 바탕으 로 비례항법 유도기법에 기반한 알고리듬을 이용 하여 표적을 추적/요격한다. 이때 김발형 적외선 탐색기(Gimballed IR Seeker)는 탐색기가 유도탄 의 동체운동과는 독립적으로 관성좌표계 상에서 표적을 지향할 수 있는 구조를 가지고 있어 비례 항법 유도명령 생성에 필요한 유도탄/표적간의 시선 각속도 정보를 직접 제공한다. 반면 탐색기 광학부를 유도탄 동체에 고정시킴으로서 정비보 수 용이, 고장수리 감소로 인한 신뢰도 증가, 단 가감소 측면 등에서 유리한 동체고정 스트랩다운 탐색기(Strapdown Seeker)에 대한 연구가 상당히 오래전부터 부분적으로 수행되어 왔다[1-3]. 최근 에는 비교적 저가의 고해상도 비냉각 검출기 제 작 기술이 발달하게 됨으로서 이를 적용한 저가 의 스트랩다운 영상탐색기에 대한 연구가 새로운 주목을 받고 있다. 그런데 동체고정 스트랩다운 탐색기에서는 비례항법에 필요한 시선변화율 (LOS Rate)이 직접주어지지 않고 탐색기 영상 평면에 맺힌 표적영상으로부터 유도탄과 표적간 의 상대운동을 추정하여 유도명령생성에 필요한 정보를 추출하여야 한다. 참고문헌[4]에서는 시선 변화율 정보를 얻기 위해 필요한 Look angle rate 추정에 $\alpha-\beta$ 필터를 적용한 기법을 제안하였 다. 참고문헌[5]에서는 저가의 스트랩다운 탐색기 와 MEMS 센서들을 언센티드 칼만필터 (Unscented Kalman Filter, UKF) 구조 내에서 결 합하는 연구를 수행하였다.

언센티드 칼만필터는 계산시간은 약간 증가하 나 확장칼만필터(Extended Kalman Filter, EKF) 에 비해 추정모델들의 강한 비선형성 등의 경우 에도 필터 정확도를 향상하고 자코비언(Jacobian) 등의 계산이 필요 없어 사용도 용이함으로 최근 에 활발한 연구가 수행되고 있다[6]. 본 연구에서 는 스트랩다운 탐색기로부터 주어지는 정보를 바 탕으로 언센티드 칼만필터(Unscented Kalman Filter, UKF)를 이용하여 유도탄과 표적간의 상대 운동을 추정하고 이 정보를 이용하여 유도명령을 생성하는 기법에 대하여 알아 보았다.

\section{ㅍ. 유도탄/표적 상대운동 추정필터}

본 절에서는 스트랩다운 탐색기 정보를 이용 하여 표적과 유도탄간의 상대적인 운동을 추정하

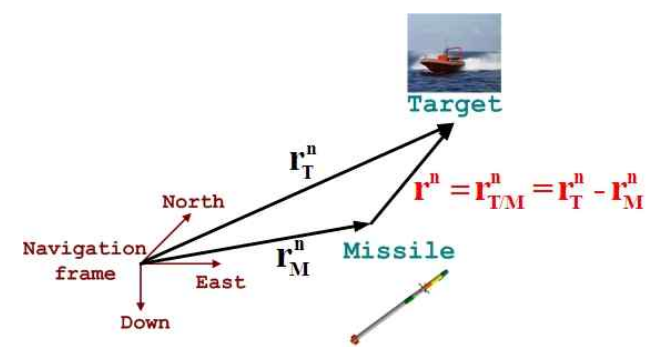

그림 1. 항법좌표계에서의 유도탄/표적 상대운동

기 위한 알고리듬에 대하여 기술한다. 먼저 스트 랩다운 탐색기 정보를 측정치로 하여 표적과 유 도탄간의 상대적인 운동을 추정할 수 있는 필터 를 설계한다. 그림 1 은 유도탄과 표적간의 상대 운동을 항법좌표계에서 표현한 것이다.

\section{1 스트랩다운 탐색기 기반의 상대운동 추정을 위한 상태공간모델}

\section{1 .1 프로세서 모델}

표적과 유도탄 간의 상대적인 운동을 추정할 수 있는 필터를 설계하기 위한 프로세서모델은 다음과 같다.

$$
\begin{gathered}
\dot{\mathbf{r}}^{n}=\mathbf{v}^{n}, \\
\dot{\mathbf{v}}^{n}=\mathbf{a}^{n}+\mathbf{n}_{a}^{n}=\mathbf{a}_{T}^{n}-\mathbf{a}_{M}^{n}+\mathbf{n}_{a}^{n}, \\
\dot{\mathbf{a}}_{T}^{n}=-\frac{1}{\tau} \mathbf{a}_{T}^{n}+\mathbf{n}_{T}^{n} .
\end{gathered}
$$

상태변수는 다음과 같이 정의하였다.

$$
\begin{gathered}
\mathbf{x}=\left[\begin{array}{c}
x_{1: 3} \\
x_{4: 6} \\
x_{7: 9}
\end{array}\right]=\left[\begin{array}{c}
\mathbf{r}^{n} \\
\mathbf{v}^{n} \\
\mathbf{a}_{T}^{n}
\end{array}\right], \\
\mathbf{r}^{n}=\mathbf{r}_{T / M}^{n}=\left[\begin{array}{c}
X \\
Y \\
Z
\end{array}\right], \mathbf{v}^{n}=\mathbf{v}_{T / M}^{n}=\left[\begin{array}{c}
U \\
V \\
W
\end{array}\right], \mathbf{a}_{T}^{n}=\left[\begin{array}{c}
a_{T x} \\
a_{T y} \\
a_{T z}
\end{array}\right] .
\end{gathered}
$$

여기서 $\mathbf{r}^{n}, \mathbf{v}^{n}$ 은 항법좌표계 상에서 나타낸 유 도탄과 표적의 상대적인 거리(그림 1) 및 속도를 나타낸다. $\mathbf{a}_{T}^{n}$ 는 1 차 Markov 프로세서 모델을 이 용하여 추정하고자 하는 표적가속도이며, 유도탄 가속도는 $\quad \mathbf{a}_{M}^{n}=\mathbf{C}_{b}^{n}(\mathbf{q}) \mathbf{a}_{M}^{b} \quad$ 로서 $\mathrm{IMU}$ 정보를 이 용한 항법소프트웨어로부터 얻어진다. $\mathbf{n}_{a}^{n}, \mathbf{n}_{T}^{n}$ 는 프로세서 노이즈 항들이며, $\mathbf{q}$ 는 유도탄의 자세 를 4 개의 파라미터로 표현하는 쿼터니언 (quaternion)이다. 


\section{1 .2 측정치 모델}

측정치 모델은 다음과 같다.

$$
\begin{aligned}
& \theta_{T}^{b}=\tan ^{-1}\left(\frac{-z_{b}}{\sqrt{x_{b}^{2}+y_{b}^{2}}}\right)+v_{\theta T}, \\
& \psi_{T}^{b}=\tan ^{-1}\left(\frac{y_{b}}{x_{b}}\right)+v_{\psi},
\end{aligned}
$$

여기서 $\theta_{T}^{b}, \psi_{T}^{b}$ 는 표적의 고각(elevation angle)과 방위각(azimuth angle)을 나타내며, $v_{\theta_{T}}, v_{\psi_{T}}$ 는 표적의 고각과 방위각의 측정노이즈를 나타낸다. $\mathbf{r}^{b}=\left[\begin{array}{lll}x_{b} & y_{b} & z_{b}\end{array}\right]^{T}=\mathbf{C}_{n}^{b} \mathbf{r}^{n}$ 의 관계가 있으며, $\mathbf{C}_{n}^{b}(\mathbf{q})$ 는 항법소프트웨어로부터 주어진다.

\subsection{3 측정치}

표적이 스트랩다운 탐색기의 이미지 평면 상 에 표시되는 개념은 그림 2에 나타나 있다. 전술 유도탄의 스트랩다운 탐색기 특성 예를 표 1에 정리하였다. 스트랩다운 탐색기의 이미지 평면 상에 표시되는 표적정보로부터 표적에 대한 고각 과 방위각을 산출하는 과정은 다음과 같다. 먼저 그림 3에 나타난 바와 같이 탐색기 이미지 평면 상의 좌표 ( ix, iy ) (단, ix $=0 \sim 639$, iy $=0$ 479)를 이용하여 이미지 평면 중심축에 대한 좌 표 $\left(y^{i}, z^{i}\right)$ 를 다음 식에 의해 구한다.

$$
\begin{aligned}
& y^{i}=i x-320 \\
& z^{i}=i y-240
\end{aligned}
$$

여기서 $y^{i}=-320 \sim 319, \quad z^{i}=-240 \sim$ 239 이다. 각 픽셀(pixel)의 ifov(instantaneous field of view)는 $\quad \frac{24^{\circ}}{640}=\frac{18^{\circ}}{480}=0.0375^{\circ}$

$=6.545 \times 10^{-4} \mathrm{rad}$ 로부터 구할 수 있다.

$$
1 \text { pixel ifov } \cong 6.545 \times 10^{-4} \mathrm{rad}
$$

\begin{tabular}{|c|c|c|}
\hline Characteristics & Data & Units \\
\hline Pixels $(\mathrm{H} \times \mathrm{V})$ & $640 \times 480$ & pixel \\
\hline$(\mathrm{H} \times \mathrm{V})$ & $24^{\circ} \times 18^{\circ}$ & degree \\
\hline
\end{tabular}

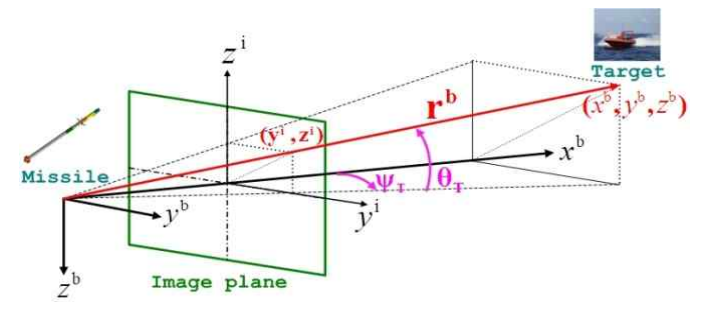

그림 2. 동체좌표계 상의 스트랩다운 탐색기 개념
표 1. 스트랩다운 탐색기 특성 예

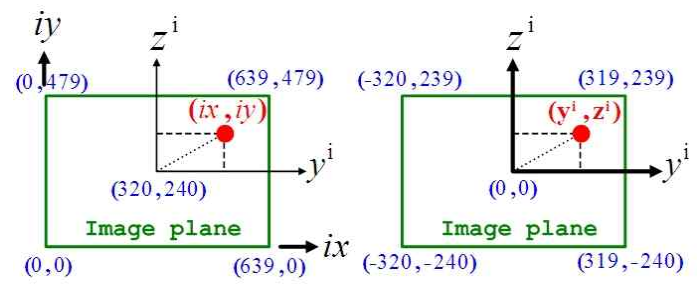

\section{그림 3. 스트랩다운 탐색기의 이미지 평면상의} 축 정의

탐색기 이미지 평면 중심축에 대한 좌표 $\left(y^{i}, z^{i}\right)$ 와 픽셀의 ifov를 이용하면 고각과 방위각을 다 음 관계식들에 의해 구할 수 있다.

$$
\begin{aligned}
& \theta_{T}^{b}=\text { ifov } * y^{i}=6.545 \times 10^{-4}(i x-320) \\
& \psi_{T}^{b}=\text { ifov } * z^{i}=6.545 \times 10^{-4}(i y-240)
\end{aligned}
$$

이 식에서 구한 $\theta_{T}^{b}, \psi_{T}^{b}$ 는 칼만필터에서 측정치 로 이용된다.

\section{2 언센티드 칼만필터}

스트랩다운 탐색기 정보를 이용하여 표적과 유도탄간의 상대운동을 추정하기 위한 필터로서 는 자코비언(Jacobian) 행렬을 계산할 필요가 없 고 비선형 필터링 성능이 우수한 $\mathrm{UKF}$ 를 사용한 다. 이 필터는 Julier 등이 최근에 개발하여 많은 주목을 받고 있으며 여러 가지 변형필터들이 Merwe 등에 의해 개발되고 있다[6,7].

상태공간 형태로 표현된 일반적인 비선형 이 산시간 프로세서 모델과 이산시간 측정모델은 다 음과 같이 주어진다.

$$
\begin{gathered}
\mathbf{x}_{k+1}=\mathbf{f}_{d}\left(\mathbf{x}_{k}, \mathbf{u}_{k}, k\right)+\mathbf{w}_{k}, \quad \mathbf{w}_{k} \sim N\left(\mathbf{0}, \mathbf{Q}_{k}\right), \\
\mathbf{y}_{k}=\mathbf{h}\left(\mathbf{x}_{k}, k\right)+\mathbf{v}_{k}, \quad \mathbf{v}_{k} \sim N\left(\mathbf{0}, \mathbf{R}_{k}\right),
\end{gathered}
$$

여기서 초기조건 $\left(\mathbf{x}\left(t_{0}\right) \sim N\left(\hat{\mathbf{x}}_{0}, \mathbf{P}_{0}\right)\right)$ 은 주어진다고 가정한다.

$\mathrm{UKF}$ 알고리듬을 적용하기 위해 주어지는 초 기조건 $\hat{\mathbf{x}}_{0}, \mathbf{S}_{0}$ 은 다음과 같다.

$$
\hat{\mathbf{x}}_{0}=E\left[\mathbf{x}\left(t_{0}\right)\right]
$$




$$
\begin{gathered}
\mathbf{P}_{0}=E\left[\left(\mathbf{x}\left(t_{0}\right)-\hat{\mathbf{x}}_{0}\right)\left(\mathbf{x}\left(t_{0}\right)-\hat{\mathbf{x}}_{0}\right)^{T}\right], \\
\mathbf{S}_{0}=\left\{\operatorname{chol}\left(\mathbf{P}_{0}\right)\right\}^{T} .
\end{gathered}
$$

Matlab 함수 "chol"은 상부 삼각(upper triangular) 형태의 Cholesky 분해(factorization) 를 구해주므로 하부 삼각(lower triangular) 형태 의 $\mathbf{S}_{0}$ 를 구하기 위해서는 행렬의 전치 (transpose)가 필요하다.

\section{A. 시간전파 (예측 단계)}

각각의 시간 간격 $k=1,2, \cdots$ 에 대하여 먼저 시그마 포인터(sigma points)를 계산하고 다음의 시간 갱신식(time-update equation)에 의하여 시 간 전파(time-propagate)가 필요하다.

- 시그마 포인트 산출

$$
\begin{gathered}
\mathbf{S}_{k-1}=\left\{\operatorname{chol}\left(\mathbf{P}_{k-1}\right)\right\}^{T}, \\
\mathbf{X}_{k-1}=\left[\begin{array}{lll}
\hat{\mathbf{x}}_{k-1} & \hat{\mathbf{x}}_{k-1}+\gamma \mathbf{S}_{k-1} & \hat{\mathbf{x}}_{k-1}-\gamma \mathbf{S}_{k-1}
\end{array}\right] .
\end{gathered}
$$

- 시간전파 갱신식

$$
\begin{gathered}
\mathbf{X}_{k \mid k-1}^{*}=\mathbf{f}_{d}\left(\mathbf{X}_{k-1}, \mathbf{u}_{k-1}\right), \\
\hat{\mathbf{x}}_{k}^{-}=\sum_{i=0}^{2 n} w_{i}^{(m)} \mathbf{X}_{i, k \mid k-1}^{*}, \\
\mathbf{P}_{k}^{-}=\sum_{i=0}^{2 n} w_{i}^{(c)}\left(\mathbf{X}_{i, k \mid k-1}^{*}-\hat{\mathbf{x}}_{k}^{-}\right)\left(\mathbf{X}_{i, k \mid k-1}^{*}-\hat{\mathbf{x}}_{k}^{-}\right)^{T}+\mathbf{Q}_{k} .
\end{gathered}
$$

단, $\mathbf{Q}_{k}=\Delta t \mathbf{Q}(t)$

B. 측정치 갱신 (교정 단계)

- 보강된 시그마 포인트 산출

$$
\begin{gathered}
\mathbf{S}_{k}^{-}=\left\{\operatorname{chol}\left(\mathbf{P}_{k}^{-}\right)\right\}^{T}, \\
\mathbf{X}_{k \mid k-1}=\left[\begin{array}{lll}
\hat{\mathbf{x}}_{k}^{-} & \hat{\mathbf{x}}_{k}^{-}+\gamma \mathbf{S}_{k}^{-} & \hat{\mathbf{x}}_{k}^{-}-\gamma \mathbf{S}_{k}^{-}
\end{array}\right], \\
\mathbf{Y}_{k \mid k-1}=\mathbf{h}\left(\mathbf{X}_{k \mid k-1}\right), \\
\hat{\mathbf{y}}_{k}^{-}=\sum_{i=0}^{2 n} w_{i}^{(m)} \mathbf{Y}_{i, k \mid k-1} .
\end{gathered}
$$

- 측정치 갱신식

$$
\begin{aligned}
& \mathbf{P}_{y}=\sum_{i=0}^{2 n} w_{i}^{(c)}\left(\mathbf{Y}_{i, k \mid k-1}-\hat{\mathbf{y}}_{k}^{-}\right)\left(\mathbf{Y}_{i, k \mid k-1}-\hat{\mathbf{y}}_{k}^{-}\right)^{T}+\mathbf{R}_{k}, \\
& \mathbf{P}_{x y}=\sum_{i=0}^{2 n} w_{i}^{(c)}\left(\mathbf{X}_{i, k \mid k-1}-\hat{\mathbf{x}}_{k}^{-}\right)\left(\mathbf{Y}_{i, k \mid k-1}-\hat{\mathbf{y}}_{k}^{-}\right)^{T},
\end{aligned}
$$

$$
\begin{gathered}
\mathbf{K}_{k}=\mathbf{P}_{x y} \mathbf{P}_{y}^{-1}, \\
\hat{\mathbf{x}}_{k}=\hat{\mathbf{x}}_{k}^{-}+\mathbf{K}_{k}\left(\mathbf{y}_{k}-\hat{\mathbf{y}}_{k}^{-}\right), \\
\mathbf{P}_{k}=\mathbf{P}_{k}^{-}-\mathbf{K}_{k} \mathbf{P}_{y} \mathbf{K}_{k}^{T} .
\end{gathered}
$$

\section{III. 스트랩다운 탐색기를 이용한 종말 유도 알고리듬}

앞 장에서 유도탄과 표적간의 상대운동에 대 한 추정결과를 토대로 다음과 같은 종말유도 알 고리듬을 설계할 수 있다[8].

$$
\begin{gathered}
\left(\mathbf{a}_{M}^{b}\right)_{c}=\mathbf{C}_{n}^{b} N\left(\frac{1}{t_{g o}^{2}} \mathbf{r}^{n}+\frac{1}{t_{g o}} \mathbf{v}^{n}+K_{T} \mathbf{a}_{T}^{n}\right), \\
K_{T}=\frac{e^{-\left(t_{g o} / \tau\right)}+\left(t_{g o} / \tau\right)-1}{\left(t_{g o} / \tau\right)^{2}}, \\
t_{g o}=\frac{2 R}{V_{c}+\sqrt{V_{c}^{2}+2 R A_{c}}}, \\
R=\left|\mathbf{r}^{n}\right|, V_{c}=\left|\mathbf{v}^{n}\right|, N=3 .
\end{gathered}
$$

$K_{T}$ 는 표적가속도 계수(Target acceleration gain)이며 $N$ 은 항법계수(navigation gain)이다.

\section{IV. 시뮬레이션 결과}

유도탄과 표적간의 상대운동을 추정하기 위한 필터의 성능을 검토해 보기 위하여 유도탄/표적 조우 시뮬레이션을 수행하고 상대운동 추정필터 를 적용하였다. 그림 4,5 는 각각 수직면 및 수평 면상의 궤적 시뮬레이션 결과를 나타낸 것이다.

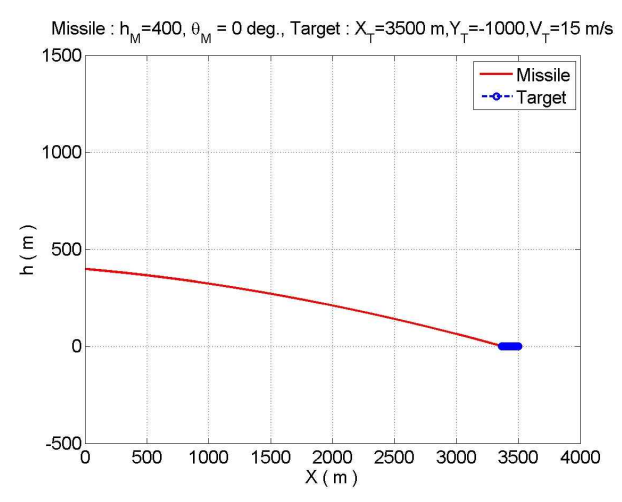

그림 4. 수직면 상의 궤적 


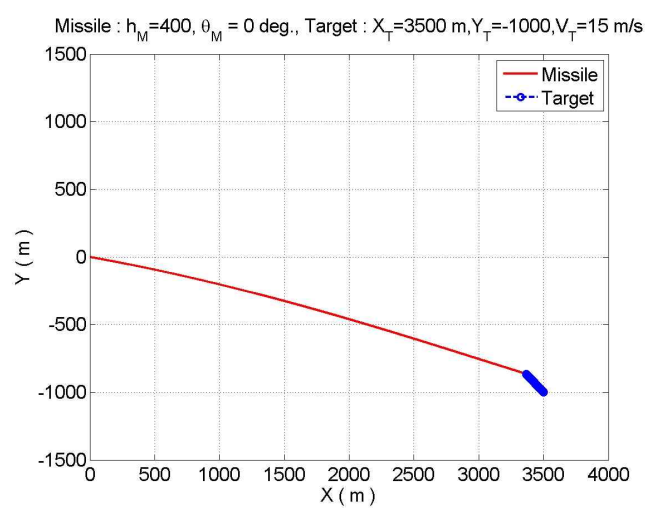

그림 5. 수평면 상의 궤적

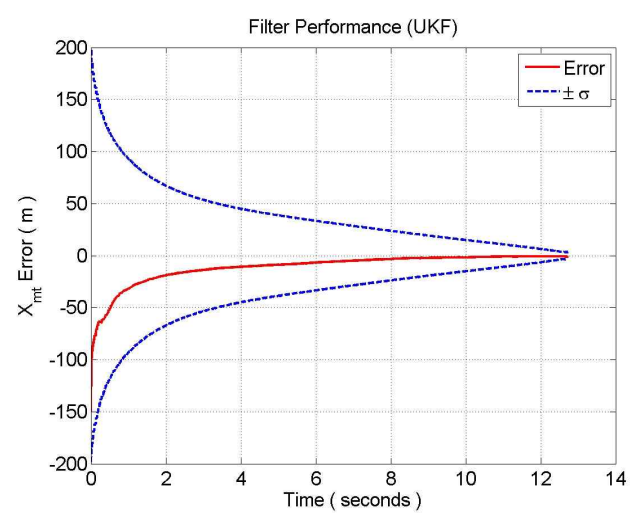

그림 6. X 방향 상대거리 오차

유도탄은 초기고도 $400 \mathrm{~m}$ (초기 방위각 : -10도, 초기 고각 : -5 도) 이며 표적의 초기위치 (3500 $\mathrm{m},-1000 \mathrm{~m}, 0 \mathrm{~m}$ )에서 방위각 135 도 방향으로 $15 \mathrm{~m} / \mathrm{sec}$ 속도로 움직인다. 표적이 해면에서 움 직이는 경우를 고려하여 표적 가속도식인 식 (3) 에서 수평면 운동만 고려하였고, 필터의 초기 오 차는 초기 위치오차 $(150 \mathrm{~m}, 150 \mathrm{~m}, 0 \mathrm{~m}$ ), 초 기 속도오차 $(5 \mathrm{~m} / \mathrm{sec}, 5 \mathrm{~m} / \mathrm{sec}, 0 \mathrm{~m} / \mathrm{sec}$ )로 가정하였다. 상대운동 추정필터의 상태변수 초기 공분산 행렬 $\mathrm{P}(0)$, 프로세서노이즈 공분산 행렬 $\mathrm{Q}$, 측정노이즈 공분산 행렬 $\mathrm{R}$ 은 각각 다음과 같이 결정하였다. $\mathrm{P}(0)=\operatorname{diag}\left\{200^{2}, 200^{2}, 200^{2}\right.$, $\left.10^{2}, 10^{2}, 10^{2}, 0.5^{2}, 0.5^{2}\right\}, \mathrm{Q}=\operatorname{diag}\left\{10^{2}, 10^{2}\right.$, $\left.10^{2}, 2^{2}, 2^{2}, 2^{2}, 0.2^{2}, 0.2^{2}\right\}, \mathrm{R}=\operatorname{diag}\left\{\left(1^{\circ}\right)^{2},\left(1^{\circ}\right)^{2}\right.$ \}. 표적가속도에 대한 1 차 Markov 모델의 시정 수 $\tau$ 는 비교적 느리게 운동하는 (lazy turn) 해 상표적을 고려하여 참고문헌[9]에 따라 $\tau=60$ 초로 정하였다.

그림 6,7 은 관성좌표계 상의 $\mathrm{X}, \mathrm{Y}$ 방향의 상 대거리 오차를 각각 필터의 $\pm 1 \sigma$ 오차 공분산 범

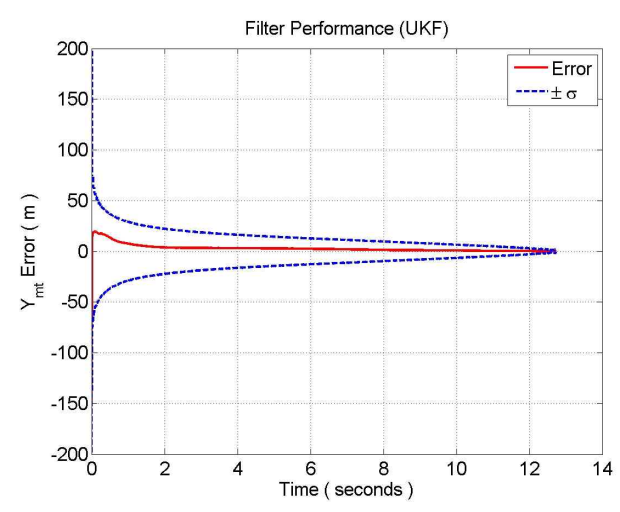

그림 7. $Y$ 방향 상대거리 오차

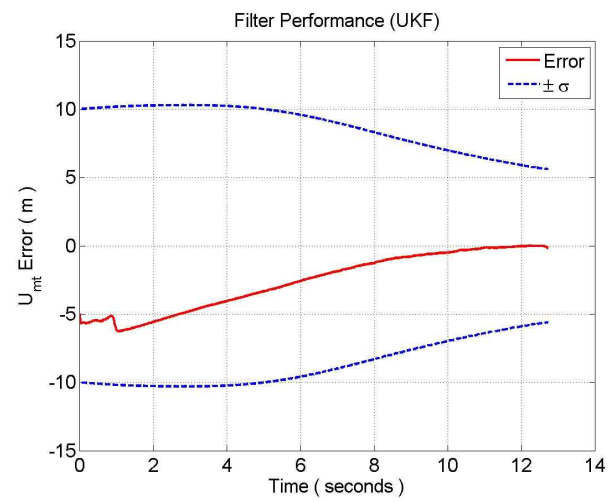

그림 8. X 방향 상대속도 $(\mathrm{U})$ 오차

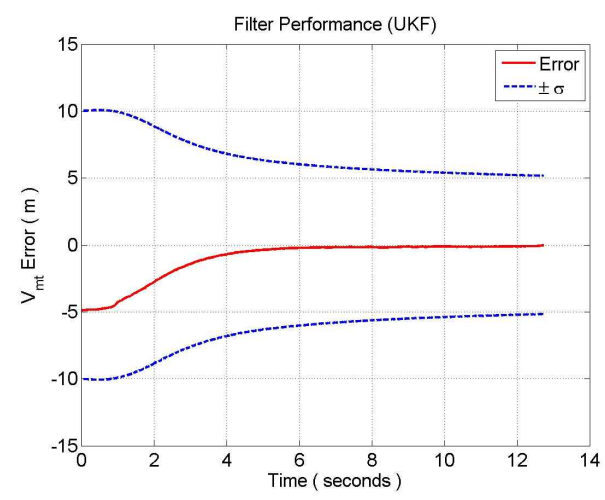

그림 9. $\mathrm{Y}$ 방향 상대속도 $(\mathrm{V})$ 오차

위와 같이 표시하였다. 각각의 초기 위치오차 $150 \mathrm{~m}$ 에서 오차크기가 $\pm 1 \sigma$ 이내에서 빠르게 오 차를 줄이며 수렴함을 확인할 수 있다. 그림 8,9 는 관성좌표계 상의 $\mathrm{X}, \mathrm{Y}$ 방향의 상대속도 오차 를 각각 필터의 오차공분산 $\pm 1 \sigma$ 범위와 같이 표 시한 것이다. 이 경우도 필터가 초기오차 5 $\mathrm{m} / \mathrm{sec}$ 에서 오차범위를 $\pm 1 \sigma$ 이내로 유지하면서 


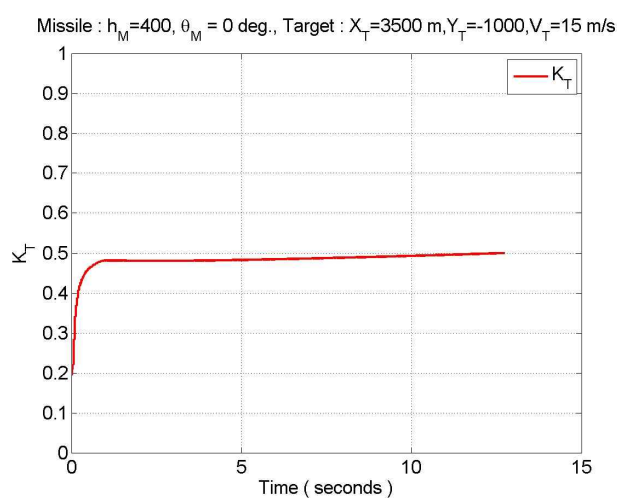

그림 10. $K_{T}$ 의 시간 변화

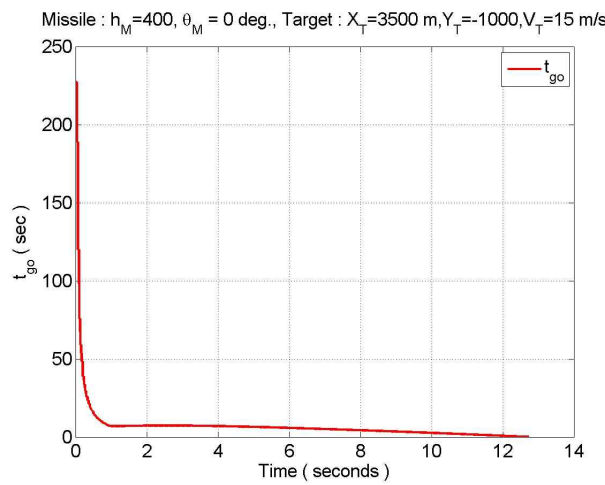

그림 11. $t_{g o}$ 의 시간 변화

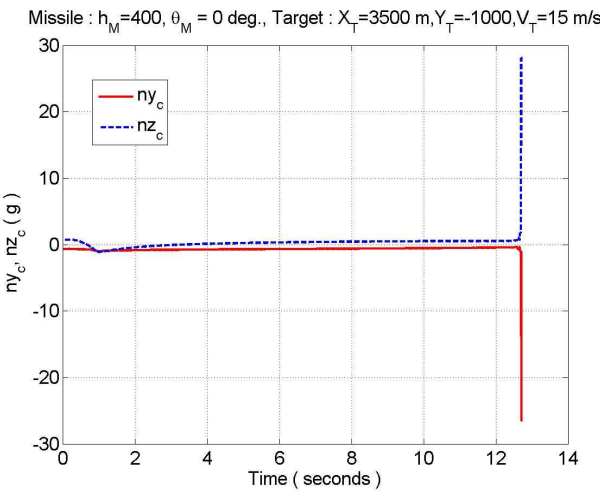

그림 12. 가속도 명령의 시간변화

수렴하는 성능을 발휘하고 있음을 알 수 있다.

그림 10,11 은 종말 유도명령 생성시 필요한

두 변수 $K_{T}, t_{g o}$ 의 시간 변화량을 나타낸다.

$K_{T}$ 값은 시간이 경과함에 따라 0.5 의 값 근처

로 수렴하고 있다. $t_{g o}$ 값은 상대운동 추정필터의

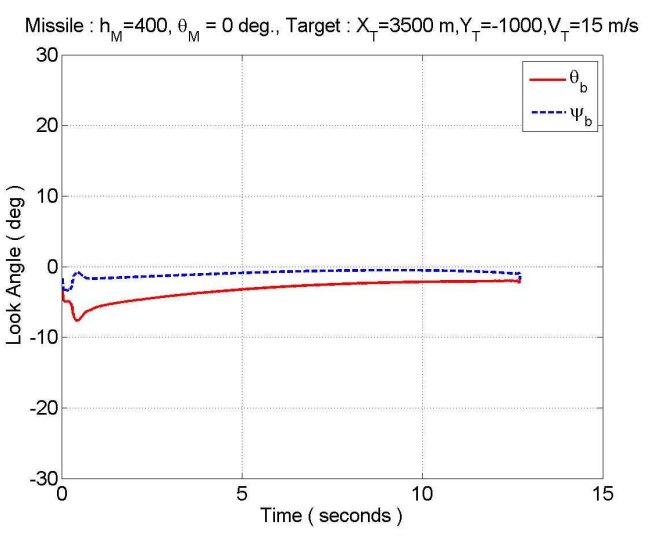

그림 13. Look Angle의 시간 변화

Missile : $h_{M}=400, \theta_{M}=0$ deg., Target $: X_{T}=3500 m, Y_{T}=-1000, V_{T}=15 \mathrm{~m} / \mathrm{s}$

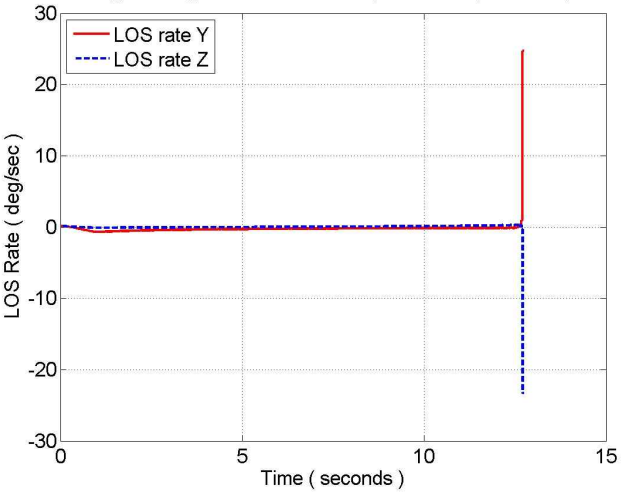

그림 14. LOS Rate의 시간 변화

여러 초기오차 요인으로 인하여 이 값도 큰 초기 오차를 보이나 필터특성이 수렴되어 감에 따라 $t_{g o}$ 값도 빠른 수렴성능을 보이고 있다.

유도탄과 표적간의 상대운동 추정필터의 여러 상태변수들과 $K_{T}, t_{g_{o}}$ 의 변수값 등을 이용하면 식(31)의 유도명령을 생성할 수 있다. 피치/요 방 향 가속도 명령으로 주어지는 유도명령의 시간변 화를 그림 12 에 나타내었다. 그림 13,14 는 각각 시간에 따른 시선각 변화와 시선 각속도 변화를 나타낸다. 시선각은 전 구간에서 10 도 이내로서 탐색기 FOV 이내임을 확인할 수 있고, 시선각속 도도 충돌직전을 제외하면 $5 \mathrm{deg} / \mathrm{sec}$ 이내이다.

\section{V. 결 론}

스트랩다운 탐색기를 적용한 전술유도탄에서 비례항법으로 유도명령을 생성하기 위해서는 유 도탄과 표적간의 상대운동을 추정하기 위한 필터 
가 필요하다. 본 연구에서 적용된 문제와 같이 강한 비선형성이 있는 경우에도 최소한 2차의 정 확도를 얻을 수 있는 $\mathrm{UKF}[6,7]$ 를 적용하여 유도 탄/표적 간의 상대운동 추정필터를 설계하고 시 뮬레이션을 통하여 성능을 검증하였다. 또한 추 정된 유도탄과 표적간의 상대운동을 바탕으로 종 말유도 알고리듬을 적용하여 유도탄/표적간의 조우운동을 시뮬레이션으로 확인할 수 있었다.

\section{후 기}

본 논문을 우리나라 초기 항공우주공학의 터 전을 마련하셨고, 저를 포함하여 비행역학 및 제 어 분야의 많은 후학들을 지도해 주신 故 위상규 (魏祥奎) 교수님의 영전에 바칩니다.

\section{참고문헌}

1) Ehrich, R. D. and Vergez, P., "Strapdown Seeker Technology for the Terminal Guidance of Tactical Weapons", AGARD Guidance and Control Aspects of Tactical Air-launched Missiles, AGARD-CP-292, N81-16092 07-15, 1980, pp. 11-1 11-15.

2) Callen, T. R., "Guidance Law Design for Tactical Weapons with Strapdown Seekers", AIAA Guidance and Control Conference, AIAA-1979-1732, Aug. 1979, pp. 281-293.

3) Vergez, P. L. and McClendon, J. R.,
"Optimal Control and Estimation for Strapdown Seeker Guidance of Tactical Missiles", J. Guidance, Vol. 5, No. 3, May-June 1982, pp. 225-226.

4) 장세아, 유창경, 최기영, 탁민제, "Guidance Algorithms for Tactical Missiles with Strapdown Seeker", SICE Annual Conference 2008, Aug. 2008, pp. 2616-2619.

5) 윤중섭, 유창경, 송택렬, “스트랩다운 탐색 기 및 $\mathrm{MEMS}$ 센서를 이용한 유도필터 설계”, 항 공우주학회지, 제37권 10호, 2009, pp. 1002-1009.

6) Julier, S. J. and Uhlmann, J. K., "A New Extension of the Kalman Filter to Nonlinear Systems", the 11th int. symposium on aerospace/defense sensing, simulation and controls, Orlando, Florida, Aug. 1997.

7) Wan, E. A. and Merwe, R. van der, "Chapter 7. The Unscented Kalman Filter", in Kalman Filtering and Neural Networks (S. HayKin ed.), New York, John Wiley \& Sons, 2001.

8) Mehra, R. K. and Ehrich, R. D., "Air-To-Air Missile Guidance for Strapdown Seekers", Proc. Of 23rd Conference on Decision And Control, Dec. 1984.

9) Li, X. R. and Jilkov, V. P., "Survey of Maneuvering Target Tracking-Part I: Dynamic Models", IEEE Trans. on Aerospace and Electronic Systems, Vol. 39, Oct. 2003, pp. 1333-1364. 